\title{
Wearable devices for patient monitoring in the early postoperative period: a literature review
}

\author{
Tajrian Amin ${ }^{1,2,3,4}$, Ralph J. Mobbs ${ }^{1,2,3,4}$, Niyaz Mostafa ${ }^{3}$, Luke W. Sy ${ }^{4,5}$, Wen Jie Choy ${ }^{1,2,3,4}$ \\ ${ }^{1}$ NeuroSpine Surgery Research Group (NSURG), Sydney, Australia; ${ }^{2}$ Neuro Spine Clinic, Prince of Wales Private Hospital, Randwick, Australia; \\ ${ }^{3}$ Faculty of Medicine, University of New South Wales (UNSW), Sydney, Australia; ${ }^{4}$ Wearables and Gait Assessment Research (WAGAR) Group, \\ Prince of Wales Private Hospital, Randwick, Australia; ${ }^{5}$ School of Biomedical Engineering, University of New South Wales, Sydney, Australia \\ Contributions: (I) Conception and design: T Amin, RJ Mobbs, WJ Choy; (II) Administrative support: T Amin, RJ Mobbs; (III) Provision of study \\ material or patients: None; (IV) Collection and assembly of data: T Amin, N Mostafa, WJ Choy; (V) Data analysis and interpretation: All authors; (VI) \\ Manuscript writing: All authors; (VII) Final approval of manuscript: All authors. \\ Correspondence to: Ralph J. Mobbs, MD, MS, FRACS. Neuro Spine Clinic, Prince of Wales Private Hospital, 320-346 Barker St., Randwick, NSW \\ 2031, Australia. Email: ralphmobbs@hotmail.com.
}

\begin{abstract}
The early postoperative period is a crucial stage in a patient's recovery as they are susceptible to a range of complications, with detection and management the key to avoiding long term consequences. Wearable devices are an innovative way of monitoring patient's post-intervention and may translate into improved patient outcomes, and reduced strain on healthcare resources, as they may facilitate safer and earlier discharge from the hospital setting. Several recent studies have investigated the use of wearable devices in postoperative monitoring. This review outlines the current literature including the range of wearable devices used for postoperative monitoring, the variety of surgeries investigated, and the outcomes assessed. A search of five electronic databases was performed. Data on the range of wearable devices, outcomes and surgeries investigated were extracted and synoptically analysed. Twenty-four articles were retrieved. Data on several different types of surgery were available and discussed. Most studies used wrist-mounted wearable devices and accelerometers or pedometers to assess physical activity metrics, including step counts and physical activity intensity (PAI), as markers of recovery. Wearable devices can provide objective data capture in the early postoperative phase to remotely monitor patients using various metrics including temperature, cardiac monitoring and physical activity. The majority of current research is focussed on wrist-mounted accelerometers and pedometers used to assess physical activity as a marker of postoperative function. Further research is required to demonstrate improved safety and cost-effectiveness of this technology.
\end{abstract}

Keywords: Wearable devices; early postoperative monitoring; physical activity monitoring; accelerometer; surface sensor technologies

Received: 19 July 2020; Accepted: 13 December 2020; Published: 20 July 2021.

doi: $10.21037 /$ mhealth-20-131

View this article at: http://dx.doi.org/10.21037/mhealth-20-131

\section{Background}

Postoperative monitoring is a vital aspect of modern surgical practice, with the aim to facilitate a fast and safe recovery. This is especially important in the early postoperative period, where multiple serious complications are prevalent $(1,2)$. Surgical insult presents a significant physiological stressor, resulting in a potent stress hormone response involving the release of catecholamines, cortisol

\footnotetext{
^ ORCID: 0000-0002-7316-1986.
} 
Table 1 Chronology of common complications of the early postoperative period (1,10-15)

\begin{tabular}{|c|c|c|}
\hline Postoperative day(s) & System & Common complications \\
\hline \multirow{4}{*}{$0-1$} & & Hypotension \\
\hline & & Arrhythmia \\
\hline & Respiratory & Respiratory depression \\
\hline & Mobility & Falls and secondary complications (fractures, head injuries, lacerations, immobility) \\
\hline \multirow[t]{5}{*}{$1-3$} & Cardiovascular & Myocardial infarction \\
\hline & & Congestive heart failure \\
\hline & & Arrhythmia \\
\hline & Genito-urinary & Urinary tract infection \\
\hline & Mobility & Falls and secondary complications (fractures, head injuries, lacerations, immobility) \\
\hline \multirow[t]{4}{*}{$4-7$} & Cardiovascular & Arrhythmia \\
\hline & Surgical site & Superficial and deep surgical site infection \\
\hline & Genito-urinary & Urinary tract infection \\
\hline & Mobility & Falls and secondary complications (fractures, head injuries, lacerations, immobility) \\
\hline
\end{tabular}

and glucagon. The subsequent systemic changes can have serious consequences (3), particularly in at-risk groups including the elderly, overweight or co-morbid (4-7). There are several common complications which may occur in the early postoperative period and can result in significant morbidity and mortality, as well as increased length of hospital stay, re-operation, readmission to hospital and significant costs $(1,8,9)$. These complications and their general chronology, in terms of peak incidence, are outlined in Table 1 (1,10-15).

Early postoperative monitoring involves the health care team regularly assessing vital signs, pain, mood, wound healing, mobility, diet, fluid balance and bowel motions. Postoperative monitoring continues post-discharge via patient education and follow up consultations with surgeons, nurses and primary care physicians $(1,2,16,17)$. There has been a recent push for early discharge and minimisation of length of hospital stay, with numerous studies reporting improvements to patient outcomes and reduced strain on hospital resources (18-23). This has included the rise of minimally invasive surgery (24-27), Enhanced Recovery After Surgery protocols (28-30) and telemedicine (31-34). The recent COVID-19 pandemic strained healthcare institutions in an unprecedented manner and further highlighted the need for innovative solutions such as telemedicine (35-38). The rise of mobile health technology has facilitated the development of medical grade wearable devices. This technology allows for a vast range of personal data to be measured by multiple potential devices and has seen widespread popularity, multiple applications and increasing sophistication (39-43). There is growing interest in the healthcare applications of wearable devices given the possibility of increasing efficiency, costeffectiveness and objectivity of collecting health-related data (39). A number of studies have investigated their use in a range of disease processes and clinical settings (44-47). One area of recent development and great promise is the application of wearable technology to early postoperative monitoring (48). As wearable devices can allow for discrete, continuous and remote collection of a range of health metrics, including cardiac monitoring, body temperature and physical activity (39), they can potentially improve the detection of complications in the early postoperative period, thereby improving patient outcomes and reducing health 


\begin{tabular}{|c|c|c|c|c|}
\hline Search & Actions & Details & Query & Results \\
\hline$\# 12$ & $\cdots$ & $>$ & $\begin{array}{l}\text { Search: }(((((\text { wearable devices) OR (wearable)) AND ((postoperative) } \\
\text { OR (intervention))) AND (monitoring)) AND (early)) AND } \\
\text { ((complications) OR (recovery)) }\end{array}$ & 47 \\
\hline$\# 11$ & $\cdots$ & $>$ & Search: (complications) OR (recovery) & $3,765,968$ \\
\hline$\# 10$ & $\cdots$ & $>$ & Search: (postoperative) OR (intervention) & $8,830,133$ \\
\hline$\# 9$ & $\cdots$ & $>$ & Search: (wearable devices) OR (wearable) & 22,958 \\
\hline$\# 8$ & $\cdots$ & $>$ & Search: recovery & 517,087 \\
\hline \#7 & $\cdots$ & $>$ & Search: complications & $3,336,664$ \\
\hline \#6 & $\cdots$ & $>$ & Search: early & $1,582,452$ \\
\hline \#5 & $\cdots$ & $>$ & Search: intervention & $8,470,145$ \\
\hline$\# 4$ & $\cdots$ & $>$ & Search: postoperative & 893,997 \\
\hline$\# 3$ & $\cdots$ & $>$ & Search: monitoring & $1,017,018$ \\
\hline$\# 2$ & $\cdots$ & $>$ & Search: wearable & 13,894 \\
\hline \#1 & ... & $>$ & Search: wearable devices & 16,170 \\
\hline
\end{tabular}

Figure 1 Structure of search and search terms for PubMed.

care costs. To our knowledge, there is no existing review of wearable devices used for early postoperative monitoring. This review of the literature looks to outline and assess the use of wearable devices for patient monitoring in the early postoperative period, with particular focus on the range of wearable devices used, the variety of surgeries investigated, and the outcomes assessed. We present the following article in accordance with the Preferred Reporting Items for Systematic Reviews and Meta-Analysis (PRISMA) reporting checklist (available at http://dx.doi.org/10.21037/ mhealth-20-131) (49).

\section{Methods}

Electronic searches were performed using Ovid Medline, PubMed, Cochrane Central Register of Controlled Trials (CCRCT), Cochrane Database of Systematic Reviews (CDSR) and Ovid Embase from their dates of inception to June 2020. To achieve the maximum sensitivity of the search strategy, we used the terms "wearable devices", "wearable", "monitoring", "postoperative", "intervention", "early", "complications" and "recovery" as either keywords or MeSH terms. The search terms were combined using Boolean operators "AND" and "OR" functions. Truncation was not used for these terms. Figure 1 outlines one of these searches. The reference lists of all full-text articles assessed for eligibility were examined to identify relevant studies as determined by the inclusion and exclusion criteria.
Two investigators independently reviewed each retrieved article (TA, NM). Discrepancies between reviewers were resolved through discussion and consensus.

\section{Inclusion criteria}

Studies eligible for this review included those in which surgical patients were monitored in the early postoperative period using wearable device technology. We defined the early postoperative period as within 7 days after surgery. All publications were limited to those involving human subjects.

\section{Exclusion criteria}

Studies including non-surgical patients were excluded. Case reports, abstracts, conference presentations, editorials, expert opinions, study protocols, patents and letters to the editor were excluded.

\section{Data collection}

All data were extracted from article texts, tables, and figures. Data of interest included the range of wearable devices investigated, the types of surgery performed, the outcomes assessed and the results produced. Two investigators independently extracted data from each retrieved article (TA, NM). Discrepancies between reviewers were resolved through discussion and consensus. 

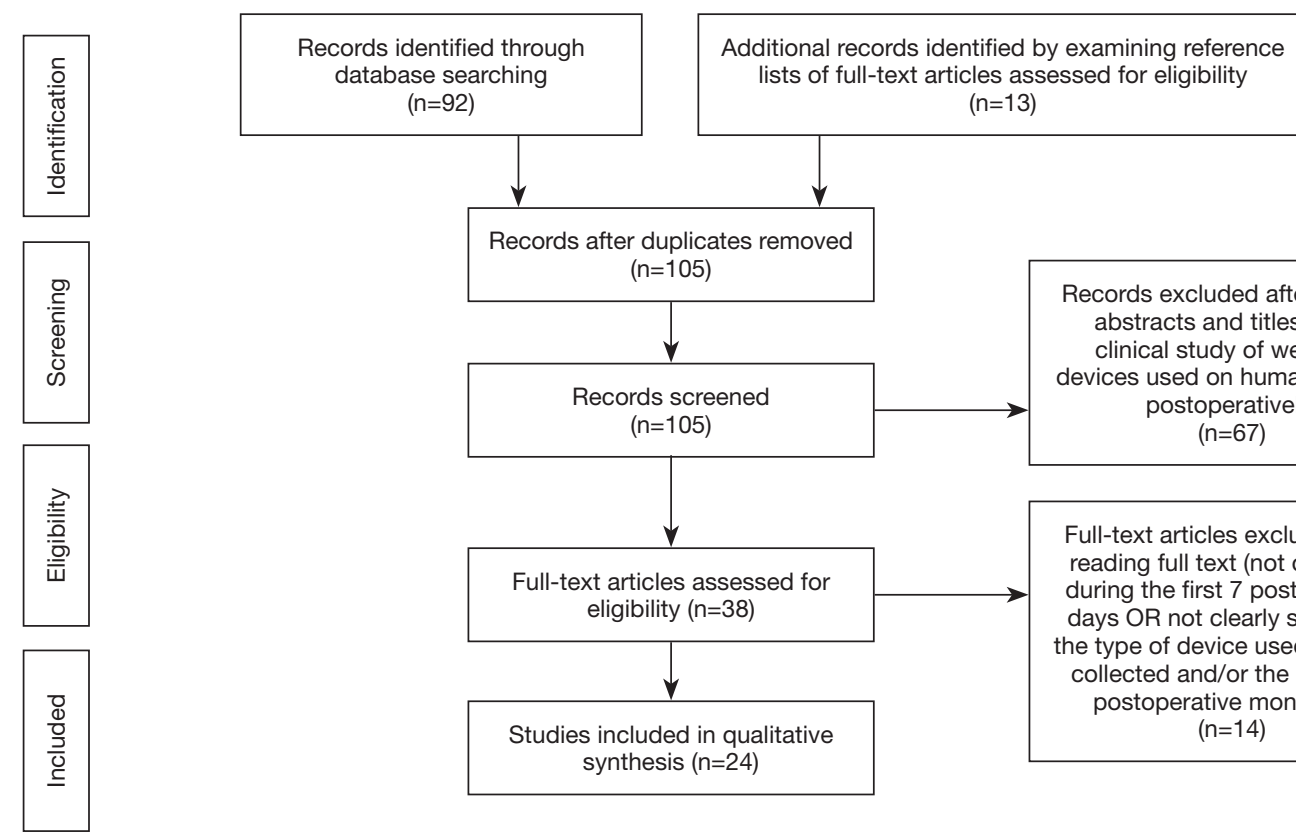
lists of full-text articles assessed for eligibility $(n=13)$

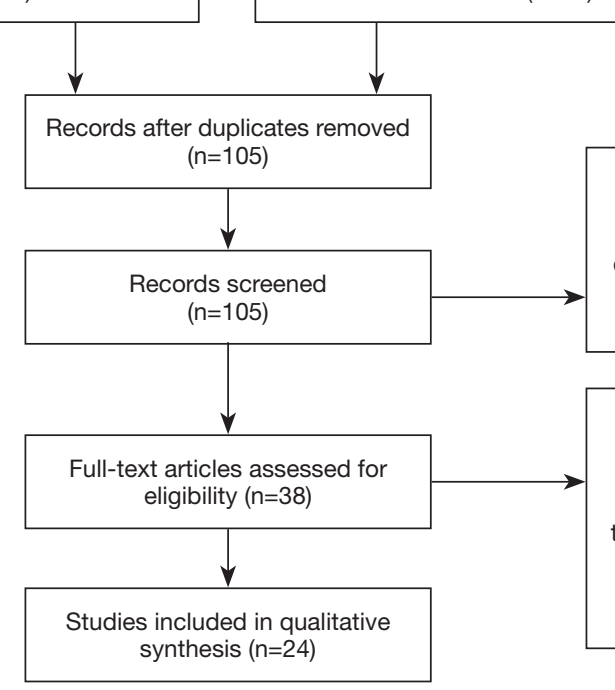
Records excluded after reading abstracts and titles (not a clinical study of wearable devices used on human patients postoperatively) $(n=67)$

Figure 2 PRISMA search strategy for the present literature review.

\section{Results}

A search of five electronic databases yielded 92 records. The inclusion and exclusion criteria were used to screen the titles and abstracts of these records, allowing for 67 articles to be excluded. The full texts of the surviving 38 articles were then assessed using the inclusion and exclusion criteria. There were 13 additional records identified in the reference lists of these articles. These were screened and surviving records also subjected to a full text analysis, in the same aforementioned manner. Ultimately 24 articles were deemed eligible and included in this review. There were six general surgery studies, five neurosurgery studies, four surgical oncology studies, two cardiac surgery studies, two orthopaedic surgery studies, two studies investigating more than one surgical specialty, one urological surgery study, one paediatric surgical study and one obstetric surgery study. The sample sizes of retrieved articles ranged from 5 to 200 subjects. A summary of the search strategy and these results can be seen in Figure 2 and Table 2, respectively.

\section{Study metrics and results}

The majority of studies identified in this review (23/24; $95.8 \%$ ) used either accelerometery or pedometery to track measures of physical activity, which served as a marker of postoperative function and recovery. Berthelot et al. (53) developed and investigated a novel wearable device which used spectroscopy to assess tissue oxygen saturation $\left(\mathrm{StO}_{2}\right)$, as a marker of free tissue transfer perfusion and survival. The most prevalent device-collected data included step counts $(17 / 24 ; 70.8 \%)$ and various measures of physical activity intensity (PAI) $(5 / 24 ; 20.8 \%)$. Other mobilityrelated data included distance ambulated $(3 / 24 ; 12.5 \%)$ measures of speed or acceleration $(4 / 24 ; 16.7 \%)$ and step length $(1 / 24 ; 4.2 \%)$. Estimates of caloric expenditure were provided by $2 / 24$ (8.3\%) studies. Agarwal et al. (50) reported sleep-related data including minutes slept and night time awakenings. Most of the retrieved articles used wrist-mounted wearable devices $(13 / 24 ; 54.2 \%)$. Other devices were ankle-mounted $(4 / 24 ; 16.7 \%)$ and waistmounted $(3 / 24 ; 12.5 \%)$. Two studies $(2 / 24 ; 8.3 \%)$ used a clip-on wearable device, with one (51) specifying where they were attached. Aziz et al. (52) used an ear-mounted device. Berthelot et al. (53) used a skin-patch type device. Fitbit, Inc. (San Francisco, CA, USA) products were investigated in 10/24 (41.7\%) studies and were the most researched devices. Several wearable devices required accessory devices $(52,55)$, an application on a mobile phone $(50,64,67,73)$, an online account, personal computer or a tablet device $(51,65,66,68,71)$ to allow for data collection and transmission. The majority of studies identified in our 
Table 2 Summary of the retrieved articles

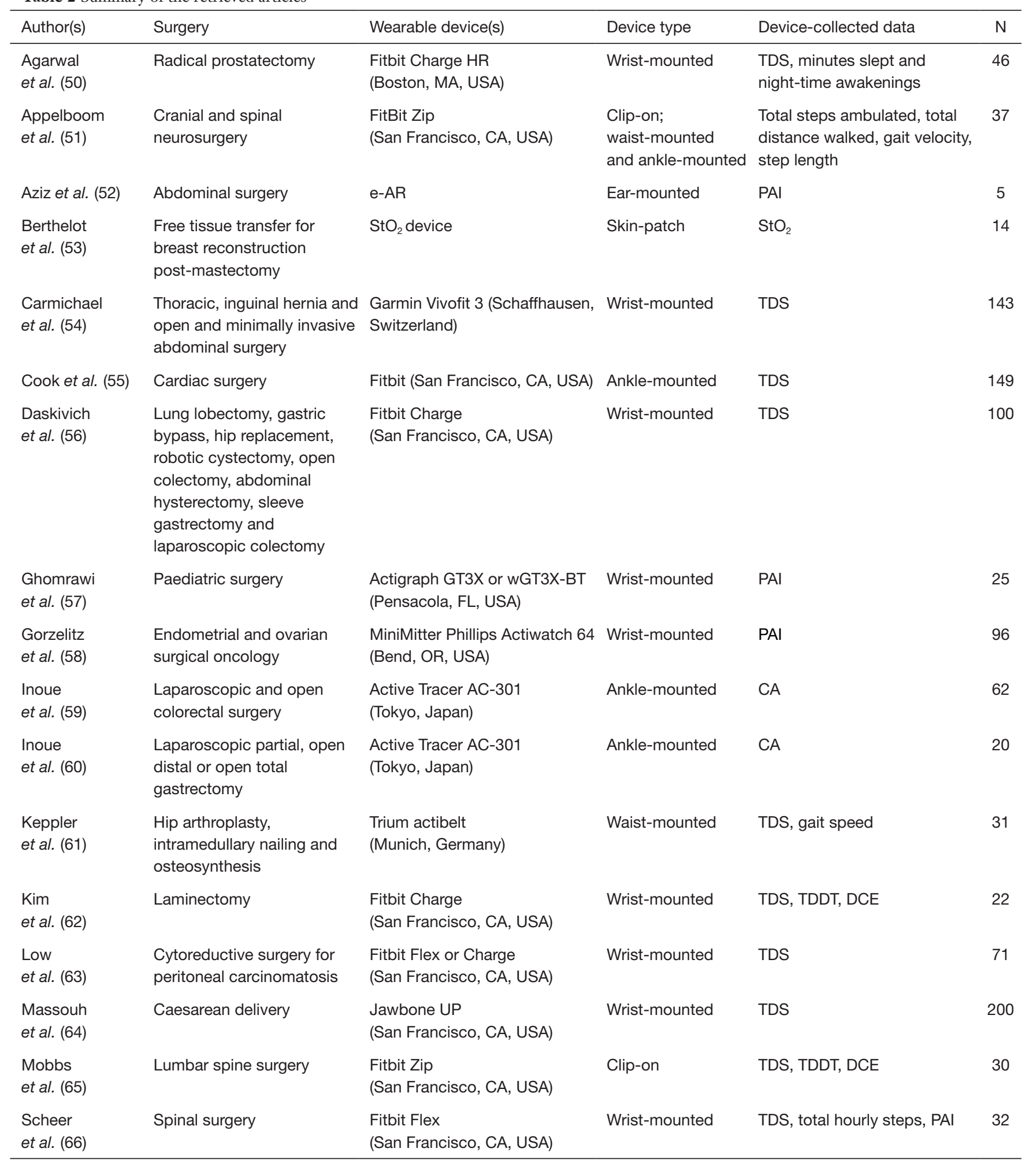

Table 2 (continued) 
Table 2 (continued)

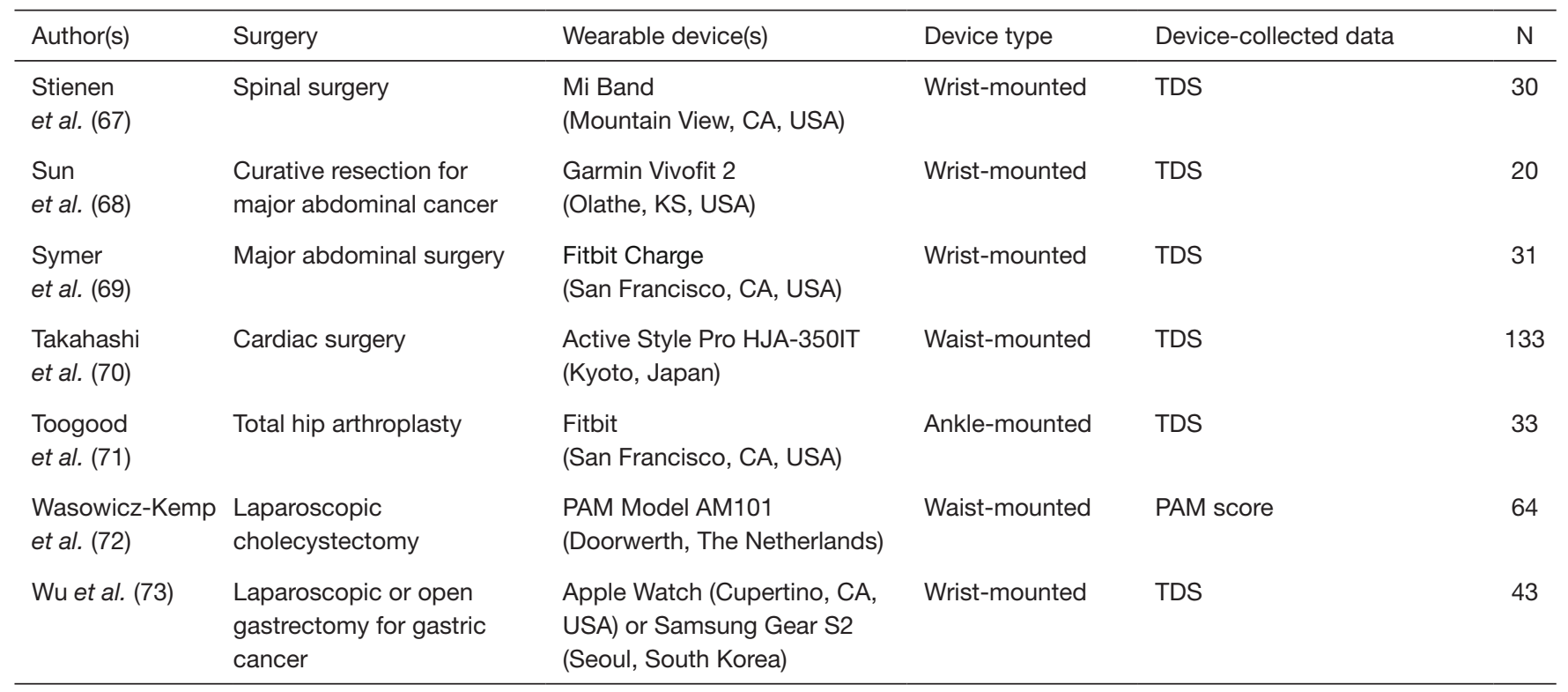

Data on surgery types, wearable devices and device-collected data is reported as presented in the retrieved articles. TDS, total daily steps; e-AR, ear-worn activity recognition; PAI, physical activity intensity (studies varied on their definitions of different intensity levels); $\mathrm{StO}_{2}$, tissue oxygen saturation; CA, cumulative acceleration; TDDT, total daily distance travelled; DCE, daily caloric expenditure; PAM, personal activity monitor.

review provide evidence to support the notion that wearable devices are useful, cost-effective and objective tools to reliably, remotely and regularly monitor postoperative function and recovery (50,53-56,59-61,63-68,71). A number of studies also reported high levels of patient compliance and satisfaction $(50,61,65,67,68,71,73)$.

\section{Discussion}

The development of wearable devices and their recent healthcare applications have led to great interest in their use in postoperative monitoring. This review of the current literature demonstrates that wearable device technology has been investigated for postoperative monitoring after a range of surgeries, with the evidence suggesting that they are a useful and objective method of continuously and remotely monitoring patients postoperatively, thereby potentially improving patient safety and reducing health care costs. The popularity of wrist-mounted devices among the retrieved articles was likely due to the pervasiveness, relatively low cost and discrete nature of watch-based sensors, such as the Fitbit products and wrist-mounted wearable devices in general. Most studies used accelerometery or pedometery to track measures of physical activity, which served as a marker of postoperative function and recovery. This was likely due to the associations between early mobilisation and improved survival and functional outcomes $(51,74-77)$. While our inclusion and exclusion criteria ensured all retrieved studies monitored patients in the early postoperative period, many studies also monitored patients preoperatively to produce a baseline to compare postoperative scores against.

\section{The potential impact of wearable devices in postoperative monitoring}

The great potential of applying wearable device technology to postoperative monitoring lies in the possibility of continuous and objective monitoring in both the hospital and the home (51), thereby improving patient safety and enhancing continuity of care. Through the continuous monitoring of multiple health metrics, potentially replacing inpatient monitoring for suitable, low-risk patients, wearable devices may facilitate earlier discharge and so reduce the length of hospital stay (52), therefore providing time and financial savings (55). These health metrics may also be quantitatively combined, potentially enhancing accuracy and allowing for more objective inter- and intrapatient comparisons. The Gait Posture index (GPi) is one 
such promising tool which demonstrates this concept by synthesising a number of physical activity metrics, ideally collected via a wearable device, into a simple index $(78,79)$. Wearable devices may also improve the implementation of prophylactic measures (62) such as tracking the body positions of patients and providing alarms after a predetermined period of inactivity to ensure that patients are rolled to prevent pressure sores. These devices may allow for the immediate detection of accidents, as well as the earlier detection of complications $(57,62)$ and patients with slow recovery trajectories, thus allowing for earlier interventions in these patient groups $(54,55,63,68,71)$. This is highlighted by Ghomrawi et al., who reported a link between a drop in mobility scores measured using wearable devices and the onset of postoperative complications, with detection occurring prior to medical attention being sought (57). Further, Mobbs et al. (80) reported a case demonstrating the utility of wearable device data, in conjunction with clinical and radiologic assessment, in detecting a postoperative complication and so allowing for timely intervention.

Specific at-risk populations identified in our review which may especially benefit from postoperative wearable device monitoring include obese patients (50), disabled patients (51) and orthogeriatric patients (61). Patient groups which may be particularly suited to wearable device monitoring include those who are difficult to adequately monitor using traditional methods. This includes patients who are elderly, young (57), intellectually impaired (68), speech impaired or who have psychiatric or substance related issues (62). However, these patients may also present unique challenges regarding informed consent, patient education and compliance, thereby necessitating a well-planned and multidisciplinary approach to ensure adequate implementation. The data provided by wearable devices may also aid the recovery process $(50,51,54,64)$. Patients may find quantitative and graphical evidence of their improvements motivating, thereby potentially creating a positive feedback loop. This can also be extended and potentially integrated into physiotherapy and rehabilitation efforts. For example, these devices may be pre-programmed to emit alarms after pre-determined periods of inactivity to ensure that newly ambulating patients mobilise frequently and progressively overload, possibly by increasing parameters such as duration, intensity or distance.

Postoperative monitoring via wearable devices may also be extended preoperatively to provide baselines for comparison, as well as form part of a prehabilitation strategy (50), thereby holistically enhancing peri-operative care. This preoperative monitoring may motivate patients to improve or maintain their function and so maximise their physiological reserve and postoperative level of function. Preoperative monitoring can also allow for the identification of lower mobility patients groups. These patients may be given more intensive prophylactic therapies to prevent immobility related complications (52). Mobbs et al. and Sun et al. both noted the discordance in scores between traditional subjective and recent objective measures, such as wearable device data, of function and recovery $(65,68)$. As such, the objective data collected by wearable devices may replace or serve as an important adjunct to static and subjective traditional monitoring tools, such as clinical assessments or patient reported questionnaires $(61,62,65-68,71)$.

\section{Limitations}

A significant issue is the lack of quality short-term and long-term data to demonstrate improved patient safety and reduced health care costs. Guidelines must also be developed to ensure responsible and ethical use of this technology. Careful assessment is required to distinguish between patients requiring in-patient monitoring and lowrisk patients suitable for safe early discharge with wearable monitoring. A desire to reduce departmental costs or to improve healthcare key performance indicators must not be allowed to sway clinical decision making. While wearable devices will likely enhance postoperative monitoring of patients, the data must still be analysed within each patient's unique clinical context. Some ultimately successful procedures may eliminate pain or serve a palliative function, yet produce minimal functional improvement, while others may provide functional improvement, but leave patients with debilitating side effects. Further, the data data themselves require require careful interpretation. Some wearable device systems may not be able to distinguish pathological movements, such as seizures, from normal ambulation, thus inappropriately over-estimating movement and possibly failing to detect a serious health event (52).

Important patient factors include the reliance of wearable devices on patient compliance, as highlighted by Ghomrawi et al. (57). Patient monitoring via wearable devices will also likely incur similar confidentiality and data safety concerns experienced by other mobile health technologies (81). These issues are particularly significant if standardisation, of outcomes, devices, data analytics or databases, is prioritised, such as for research purposes. Further, raw data, such as 
acceleration, may still violate patient privacy given that unknown diseases or personal habits may be detected. Robust protections, such as data encryption, password protection and access restrictions, must be employed to provide safety and patients must also be educated in order to alleviate their concerns $(50,55)$. Some patients may also find quantitative and graphical evidence of slow recovery, plateauing or regression demotivating, thereby potentially creating a negative feedback loop. To ensure efficacy, promote compliance and improve the lifespan of wearable devices patients must also be educated on how to use and maintain their devices.

Wearable device factors include the set-up, maintenance, repair and educations costs associated with these devices. These may be substantial and are a significant institutional barrier to uptake and will likely ultimately be borne by patients, governments or both. Preoperative monitoring further increases the cost of the system and may be deemed wasteful in the event of cancelled or delayed procedures. Crucially, some studies have questioned the accuracy of the data provided by wearable devices. Appelboom et al. reported that wearable devices tended to be more accurate for healthier and more mobile patients, whereas patients with slow and minimised movements as well as those using aids such as walkers had their movements underestimated (51). Some studies have indicated that accelerometers may fail to register very fast movements (71) and that wearable devices may consistently inaccurately assess step counts and PAI $(64,82)$. Also, there is a paucity of research into inter-device reliability for many of the commercially available wearable device brands, as well as into intra-device reliability (82). Some devices may require frequent charging and syncing of data, which may impair compliance and data capture $(50,73)$. Additional problems include device malfunction, damage or loss $(56,57,66)$. Protocols must be developed to rapidly assess and respond to device failure, significant patient non-compliance or faulty readings. These issues raise the potential time and financial costs associated with the technology. Also, most devices identified in this review were wrist-mounted, likely due to the pervasiveness and discretion of existing wrist-mounted wearable devices. Ankle-mounted or ear-mounted devices may therefore experience issues due to their size, anatomical location and lack of public familiarity.

\section{Limitations of the research}

Research into the healthcare applications of wearable devices is a nascent area. In the niche of postoperative monitoring, there is an abundance of pilot studies, validation studies and studies with small sample sizes. It is difficult to compare the studies in this review meaningfully and directly due to significant inter-study variation, including in devices, study time periods, frequency of monitoring, outcomes, and samples examined by different studies. Some studies did not fully report the exact device model used, their methodology or their results. Several studies did not report the actual data recorded by their wearable device or how data were manipulated, only a study-specific outcome. This may reflect protections placed on commercially available wearable devices to safeguard intellectual property, such as the methodology of computing input data in order to produce output data. One study incentivised participation by providing gift cards to subjects, thereby impairing the external validity of their results (57). Further, patients undergoing surgery due to cancer may have also received chemotherapy and/or radiotherapy, and as such are no longer solely surgical patients.

\section{Further research}

There is an urgent need for further research to substantiate the potential of this technology, most importantly to demonstrate improved patient and institutional outcomes. Ideally large, multi-centre prospective trials should be run, using validated tools to assess outcomes and therefore demonstrate the safety and cost-effectiveness of this technology. The financial analysis may involve comparisons of costs associated with beginning and maintaining wearable device monitoring programs against savings made due to reduced staffing needs, complication rates and hospital lengths of stay. The value of longer-term postoperative monitoring may also be assessed, possibly through discrete devices or by using wearable devices already used by consumers, which may additionally enhance patient compliance. This is particularly important given the findings of Gorzelitz et al. (58) and Carmichael et al. (54) who reported impaired activity levels at 4 months and at 28 days postoperatively, respectively. However, the specificity of their relatively well-educated, wealthy female cohort with gynaecologic cancer limits their external validity. Also, a lack of a preoperative baseline renders a comparison with postoperative values impossible.

While wearable devices may be useful tools for postoperative monitoring for any surgery or procedure, some devices and data may be more useful for certain 
surgeries. For example, a wearable device with sensors on either side of a joint may be be a useful tool to monitor changes in joint range of motion following orthopaedic surgery. This is highlighted by Appelboom et al. demonstrating that hip-mounted sensors were more accurate in detecting movement than ankle-mounted sensors (51). Research is needed to thus optimise devices, their application and the outcomes assessed for different surgical specialties and surgeries. Ideally, cost-benefit analyses should also be performed to balance increased number or sophistication of sensors and the value of resultant information against costs and patient comfort. Research is also needed to validate the length of preoperative monitoring needed for a significant baseline to be developed. This may vary depending on patient factors such as American Society of Anaesthesiologists (ASA) Classifications (83) and surgical factors such as the type of surgery. Research into the value of postoperative monitoring data alone is still needed, as this may be useful in monitoring emergency or preoperatively non-compliant patients.

While data trends are generally thought to be useful, researchers may also determine whether demographicmatched absolute data values, for example by age, gender and ASA Classifications, are useful as well. Research is also needed to define significant drops in collected data in order to warrant patient contact or emergency intervention (57), as well as to define thresholds or guidelines for objective input into discharge planning (55). While much of the research identified in this review is focussed on physical activity, the feasibility of collecting other types of data, such as vital signs, and their usefulness should be explored. These data types may ultimately be synthesised into an index capable of quantitatively representing overall patient function, thereby making greater use of currently available health data and extending existing tools, such as the GPi $(78,79)$, to more accurately assess and monitor patient recovery.

\section{Conclusions}

In summation, wearable devices are a promising innovative approach to postoperative patient monitoring, with the potential to remotely assess a range of health metrics and so provide objective outcomes assessment, monitor and detect early complications, improve patient safety and potentially reduce healthcare costs. The current literature is focussed on wrist-mounted accelerometers and pedometers used to assess physical activity as a marker of postoperative function and recovery, however a range of wearable options will likely evolve with more detailed data streams to improve every aspect of postoperative care. These studies suggest that wearable devices are feasible and cost-effective tools capable of remotely and regularly monitoring patients postoperatively. Further research is required to validate these findings and develop this nascent field.

\section{Acknowledgments}

The NeuroSpine Surgery Research Group (NSURG) aided with manuscript production. The Wearables and Gait Assessment Research (WAGAR) Group provided assistance with wearables literature. The Neuro Spine Clinic provided clinic, provided clinic assistance for authors.

Funding: None.

\section{Footnote}

Reporting Checklist: The authors have completed the PRISMA reporting checklist. Available at http://dx.doi. org/10.21037/mhealth-20-131

Conflicts of Interest: All authors have completed the ICMJE uniform disclosure form (available at http://dx.doi. org/10.21037/mhealth-20-131). The authors have no conflicts of interest to declare.

Ethical Statement: The authors are accountable for all aspects of the work in ensuring that questions related to the accuracy or integrity of any part of the work are appropriately investigated and resolved.

Open Access Statement: This is an Open Access article distributed in accordance with the Creative Commons Attribution-NonCommercial-NoDerivs 4.0 International License (CC BY-NC-ND 4.0), which permits the noncommercial replication and distribution of the article with the strict proviso that no changes or edits are made and the original work is properly cited (including links to both the formal publication through the relevant DOI and the license). See: https://creativecommons.org/licenses/by-nc-nd/4.0/.

\section{References}

1. Thompson JS, Baxter BT, Allison JG, et al. Temporal patterns of postoperative complications. Arch Surg 2003;138:596-602; discussion 602-3. 
2. Williams NS, Bulstrode CJK, O'Connell PR. Bailey \& Love's short practice of surgery. 25th ed. Boca Raton: CRC Press, 2018.

3. Moor D, Aggarwal G, Quiney N. Systemic response to surgery. Surgery (Oxford) 2017;35:220-3.

4. Seeliger H, Christians S, Angele MK, et al. Risk factors for surgical complications in distal pancreatectomy. Am J Surg 2010;200:311-7.

5. Shibata C, Ogawa H, Nakano T, et al. Influence of age on postoperative complications especially pneumonia after gastrectomy for gastric cancer. BMC Surg 2019;19:106.

6. Sun HC, Qin LX, Wang L, et al. Risk factors for postoperative complications after liver resection. Hepatobiliary Pancreat Dis Int 2005;4:370-4.

7. Ito T, Maekawa H, Sakurada M, et al. Risk factors for postoperative complications in patients on maintenance hemodialysis who undergo abdominal surgery. Asian J Surg 2016;39:211-7.

8. Morris MS, Deierhoi RJ, Richman JS, et al. The relationship between timing of surgical complications and hospital readmission. JAMA Surg 2014;149:348-54.

9. Bosma E, Veen EJ, de Jongh MAC, et al. Variable impact of complications in general surgery: a prospective cohort study. Can J Surg 2012;55:163-70.

10. Glaysher MA, Cresswell AB. Management of common surgical complications. Surgery (Oxford) 2017;35:190-4.

11. Hyder JA, Wakeam E, Arora V, et al. Investigating the "Rule of W," a mnemonic for teaching on postoperative complications. J Surg Educ 2015;72:430-7.

12. Kronzer VL, Jerry MR, Ben Abdallah A, et al. Preoperative falls predict postoperative falls, functional decline, and surgical complications. EBioMedicine 2016;12:302-8.

13. Lam CF, Hsieh SY, Wang JH, et al. Incidence and characteristic analysis of in-hospital falls after anesthesia. Perioper Med (Lond) 2016;5:11.

14. Church S, Robinson TN, Angles EM, et al. Postoperative falls in the acute hospital setting: characteristics, risk factors, and outcomes in males. Am J Surg 2011;201:197-202.

15. Jørgensen CC, Kehlet H; Lundbeck Foundation Centre for Fast-track Hip and Knee Replacement Collaborative Group. Fall-related admissions after fast-track total hip and knee arthroplasty - cause of concern or consequence of success? Clin Interv Aging 2013;8:1569-77.

16. Doherty GM. Current diagnosis \& treatment: surgery. 15th ed. New York: McGraw-Hill Companies, Inc., 2015.

17. Hughes E. Principles of post-operative patient care. Nurs Stand 2004;19:43-51; quiz 53.

18. Regenbogen SE, Cain-Nielsen AH, Norton EC, et al.
Costs and consequences of early hospital discharge after major inpatient surgery in older adults. JAMA Surg 2017;152:e170123.

19. Pajarón-Guerrero M, Fernández-Miera MF, DueñasPuebla JC, et al. Early discharge programme on hospital-at-home evaluation for patients with immediate postoperative course after laparoscopic colorectal surgery. Eur Surg Res 2017;58:263-73.

20. Marfil-Garza BA, Belaunzarán-Zamudio PF, GuliasHerrero A, et al. Risk factors associated with prolonged hospital length-of-stay: 18-year retrospective study of hospitalizations in a tertiary healthcare center in Mexico. PLoS One 2018;13:e0207203.

21. Williams TA, Ho KM, Dobb GJ, et al. Effect of length of stay in intensive care unit on hospital and long-term mortality of critically ill adult patients. Br J Anaesth 2010;104:459-64.

22. Krell RW, Girotti ME, Dimick JB. Extended length of stay after surgery: complications, inefficient practice, or sick patients? JAMA Surg 2014;149:815-20.

23. Bueno H, Ross JS, Wang Y, et al. Trends in length of stay and short-term outcomes among medicare patients hospitalized for heart failure, 1993-2006. JAMA 2010;303:2141-7.

24. Jaschinski T, Mosch CG, Eikermann M, et al. Laparoscopic versus open surgery for suspected appendicitis. Cochrane Database Syst Rev 2018;11:CD001546.

25. Bhave Chittawar P, Franik S, Pouwer AW, et al. Minimally invasive surgical techniques versus open myomectomy for uterine fibroids. Cochrane Database Syst Rev 2014;(10):CD004638.

26. Iribarne A, Easterwood R, Chan EYH, et al. The golden age of minimally invasive cardiothoracic surgery: current and future perspectives. Future Cardiol 2011;7:333-46.

27. Treuting R. Minimally invasive orthopedic surgery: arthroscopy. Ochsner J 2000;2:158-63.

28. Melnyk M, Casey RG, Black P, et al. Enhanced recovery after surgery (ERAS) protocols: time to change practice? Can Urol Assoc J 2011;5:342-8.

29. Varadhan KK, Neal KR, Dejong CHC, et al. The enhanced recovery after surgery (ERAS) pathway for patients undergoing major elective open colorectal surgery: A meta-analysis of randomized controlled trials. Clin Nutr 2010;29:434-40.

30. Ljungqvist $O$, Scott $M$, Fearon KC. Enhanced recovery after surgery: a review. JAMA Surg 2017;152:292-8.

31. Asiri A, AlBishi S, AlMadani W, et al. The use of telemedicine in surgical care: a systematic review. Acta 
Inform Med 2018;26:201-6.

32. Armaignac DL, Saxena A, Rubens M, et al. Impact of telemedicine on mortality, length of stay, and cost among patients in progressive care units: experience from a large healthcare system. Crit Care Med 2018;46:728-35.

33. Gunter RL, Chouinard S, Fernandes-Taylor S, et al. Current use of telemedicine for post-discharge surgical care: a systematic review. J Am Coll Surg 2016;222:915-27.

34. Greenup EP, McCusker M, Potts BA, et al. The efficacy of telemedicine-supported discharge within an in home model of care. Telemed J E Health 2017;23:763-5.

35. Ohannessian R, Duong TA, Odone A. Global telemedicine implementation and integration within health systems to fight the COVID-19 pandemic: a call to action. JMIR Public Health Surveill 2020;6:e18810.

36. Huang C, Wang Y, Li X, et al. Clinical features of patients infected with 2019 novel coronavirus in Wuhan, China. Lancet 2020;395:497-506.

37. Hollander JE, Carr BG. Virtually Perfect? Telemedicine for Covid-19. N Engl J Med 2020;382:1679-81.

38. Rees EM, Nightingale ES, Jafari Y, et al. COVID-19 length of hospital stay: a systematic review and data synthesis. BMC Med 2020;18:270.

39. Steinhubl SR, Muse ED, Topol EJ. The emerging field of mobile health. Sci Transl Med 2015;7:283rv3.

40. Peake JM, Kerr G, Sullivan JP. A critical review of consumer wearables, mobile applications, and equipment for providing biofeedback, monitoring stress, and sleep in physically active populations. Front Physiol 2018;9:743.

41. Haghi M, Thurow K, Stoll R. Wearable devices in medical internet of things: scientific research and commercially available devices. Healthc Inform Res 2017;23:4-15.

42. Seneviratne S, Hu Y, Nguyen T, et al. A survey of wearable devices and challenges. IEEE Commun Surv Tutor 2017;19:2573-620.

43. Yang H, Yu J, Zo H, et al. User acceptance of wearable devices: an extended perspective of perceived value. Telemat Inform 2016;33:256-69.

44. Noorkõiv M, Rodgers H, Price CI. Accelerometer measurement of upper extremity movement after stroke: a systematic review of clinical studies. J Neuroeng Rehabil 2014;11:144.

45. Culhane KM, O'Connor M, Lyons D, et al. Accelerometers in rehabilitation medicine for older adults. Age Ageing 2005;34:556-60.

46. Metcalf D, Milliard STJ, Gomez M, et al. Wearables and the internet of things for health: wearable, interconnected devices promise more efficient and comprehensive health care. IEEE Pulse 2016;7:35-9.

47. Berlin JE, Storti KL, Brach JS. Using activity monitors to measure physical activity in free-living conditions. Phys Ther 2006;86:1137-45.

48. Appelboom G, Yang AH, Christophe BR, et al. The promise of wearable activity sensors to define patient recovery. J Clin Neurosci 2014;21:1089-93.

49. Moher D, Liberati A, Tetzlaff J, et al. Preferred reporting items for systematic reviews and meta-analyses: the PRISMA statement. PLoS Med 2009;6:e1000097.

50. Agarwal DK, Viers BR, Rivera ME, et al. Physical activity monitors can be successfully implemented to assess perioperative activity in urologic surgery. Mhealth 2018;4:43.

51. Appelboom G, Taylor BE, Bruce E, et al. Mobile phoneconnected wearable motion sensors to assess postoperative mobilization. JMIR Mhealth Uhealth 2015;3:e78.

52. Aziz O, Atallah L, Lo B, et al. Ear-worn body sensor network device: an objective tool for functional postoperative home recovery monitoring. J Am Med Inform Assoc 2011;18:156-9.

53. Berthelot M, Henry FP, Hunter J, et al. Pervasive wearable device for free tissue transfer monitoring based on advanced data analysis: clinical study report. J Biomed Opt 2019;24:1-8.

54. Carmichael H, Overbey DM, Hosokawa P, et al. Wearable technology-a pilot study to define "normal" postoperative recovery trajectories. J Surg Res 2019;244:368-73.

55. Cook DJ, Thompson JE, Prinsen SK, et al. Functional recovery in the elderly after major surgery: assessment of mobility recovery using wireless technology. Ann Thorac Surg 2013;96:1057-61.

56. Daskivich TJ, Houman J, Lopez M, et al. Association of wearable activity monitors with assessment of daily ambulation and length of stay among patients undergoing major surgery. JAMA Netw Open 2019;2:e187673.

57. Ghomrawi HMK, Baumann LM, Kwon S, et al. Using accelerometers to characterize recovery after surgery in children. J Pediatr Surg 2018;53:1600-5.

58. Gorzelitz J, Costanzo ES, Spencer RJ, et al. Longitudinal assessment of post-surgical physical activity in endometrial and ovarian cancer patients. PLoS One 2019;14:e0223791.

59. Inoue Y, Kimura T, Noro H, et al. Is laparoscopic colorectal surgery less invasive than classical open surgery? Quantitation of physical activity using an accelerometer to assess postoperative convalescence. Surg Endosc 2003;17:1269-73. 
60. Inouez Y, Kimura T, Fujita S, et al. A new parameter for assessing postoperative recovery of physical activity using an accelerometer. Surg Today 2003;33:645-50.

61. Keppler AM, Holzschuh J, Pfeufer D, et al. Postoperative physical activity in orthogeriatric patients - new insights with continuous monitoring. Injury 2020;51:628-32.

62. Kim DH, Nam KH, Choi BK, et al. The usefulness of a wearable device in daily physical activity monitoring for the hospitalized patients undergoing lumbar surgery. J Korean Neurosurg Soc 2019;62:561-6.

63. Low CA, Bovbjerg DH, Ahrendt S, et al. Fitbit step counts during inpatient recovery from cancer surgery as a predictor of readmission. Ann Behav Med 2018;52:88-92.

64. Massouh F, Martin R, Chan B, et al. Is activity trackermeasured ambulation an accurate and reliable determinant of postoperative quality of recovery? A prospective cohort validation study. Anesth Analg 2019;129:1144-52.

65. Mobbs RJ, Phan K, Maharaj M, et al. Physical activity measured with accelerometer and self-rated disability in lumbar spine surgery: a prospective study. Global Spine J 2016;6:459-64.

66. Scheer JK, Bakhsheshian J, Keefe MK, et al. Initial experience with real-time continuous physical activity monitoring in patients undergoing spine surgery. Clin Spine Surg 2017;30:E1434-43.

67. Stienen MN, Rezaii PG, Ho AL, et al. Objective activity tracking in spine surgery: a prospective feasibility study with a low-cost consumer grade wearable accelerometer. Sci Rep 2020;10:4939.

68. Sun V, Dumitra S, Ruel N, et al. Wireless monitoring program of patient-centered outcomes and recovery before and after major abdominal cancer surgery. JAMA Surg 2017;152:852-9.

69. Symer MM, Abelson JS, Milsom J, et al. A mobile health application to track patients after gastrointestinal surgery: results from a pilot study. J Gastrointest Surg 2017;21:1500-5.

70. Takahashi T, Kumamaru M, Jenkins S, et al. In-patient step count predicts re-hospitalization after cardiac surgery. J Cardiol 2015;66:286-91.

71. Toogood PA, Abdel MP, Spear JA, et al. The monitoring of activity at home after total hip arthroplasty. Bone Joint J 2016;98-B:1450-4.

72. Wasowicz-Kemps DK, Slootmaker SM, Kemps HMC, et al. Resumption of daily physical activity after daycase laparoscopic cholecystectomy. Surg Endosc 2009;23:2034-40.
73. Wu JM, Ho TW, Chang YT, et al. Wearable-based mobile health app in gastric cancer patients for postoperative physical activity monitoring: focus group study. JMIR Mhealth Uhealth 2019;7:e11989.

74. Macchi C, Fattirolli F, Lova RM, et al. Early and late rehabilitation and physical training in elderly patients after cardiac surgery. Am J Phys Med Rehabil 2007;86:826-34.

75. Cumming TB, Thrift AG, Collier JM, et al. Very early mobilization after stroke fast-tracks return to walking: further results from the phase II AVERT randomized controlled trial. Stroke 2011;42:153-8.

76. Grass F, Pache B, Martin D, et al. Feasibility of early postoperative mobilisation after colorectal surgery: a retrospective cohort study. Int J Surg 2018;56:161-6.

77. de Almeida EPM, de Almeida JP, Landoni G, et al. Early mobilization programme improves functional capacity after major abdominal cancer surgery: a randomized controlled trial. Br J Anaesth 2017;119:900-7.

78. Ghent F, Mobbs RJ, Mobbs RR, et al. Assessment and Post-Intervention recovery following surgery for Lumbar Disc Herniation based on objective gait metrics from wearable devices using the Gait Posture index: GPi ${ }^{\mathrm{TM}}$. World Neurosurg 2020;142:e111-6.

79. Mobbs RJ, Mobbs RR, Choy WJ. Proposed objective scoring algorithm for assessment and intervention recovery following surgery for lumbar spinal stenosis based on relevant gait metrics from wearable devices: the Gait Posture index (GPi). J Spine Surg 2019;5:300-9.

80. Mobbs RJ, Katsinas CJ, Choy WJ, et al. Objective monitoring of activity and Gait Velocity using wearable accelerometer following lumbar microdiscectomy to detect recurrent disc herniation. J Spine Surg 2018;4:792-7.

81. Arora S, Yttri J, Nilse W. Privacy and security in mobile health (mHealth) research. Alcohol Res 2014;36:143-51.

82. Evenson KR, Goto MM, Furberg RD. Systematic review of the validity and reliability of consumer-wearable activity trackers. Int J Behav Nutr Phys Act 2015;12:159.

83. Doyle DJ, Goyal A, Bansal P, et al. American Society of Anesthesiologists classification (ASA class). In: StatPearls [Internet]. Treasure Island: StatPearls Publishing, 2020.

doi: $10.21037 /$ mhealth-20-131

Cite this article as: Amin T, Mobbs RJ, Mostafa N, Sy LW, Choy WJ. Wearable devices for patient monitoring in the early postoperative period: a literature review. mHealth 2021;7:50. 